\title{
Introduction to the Special Issue: Teacher Educators for Children with Behavioral Disorders (TECBD) Conference
}

\author{
Sarup R. Mathur $(\mathbb{D} \cdot$ Wendy Peia Oakes $(\mathbb{D} \cdot$ Eric Alan \\ Common (i) - Rebecca E. Hartzell (i)
}

Published online: 9 September 2021

(C) Association for Behavior Analysis International 2021

COVID-19 has yielded extraordinary challenges and profound disruptions to traditional academic research. The 2020 annual Teacher Educators of Children with Behavioral Disorders (TECBD) Conference, hosted by Arizona State University, was held virtually. Although we had a reduced number of submissions this year, the high quality of them allowed us to maintain the tradition of producing this special issue. For this special issue of ETC, we offer five articles selected using on a doubleblind review process. The first two articles relate to tiered systems of supports and decision making. The next two articles focus on specific interventions, selfregulated strategy development and precision request, and their use with students with emotional and behavioral disorders (EBD). The final article presents findings of a survey that assessed educators' perceived trust and use of classroom behavior management resources. We hope that readers find the content of this special issue interesting, enlightening, and relevant on their quest for knowledge. We applaud the authors for their persistence

S. R. Mathur $(\bowtie) \cdot$ W. P. Oakes

Mary Lou Fulton Teachers College, Arizona State University, Farmer 408 C, Arizona State University, Tempe, AZ, USA e-mail: Sarup.Mathur@asu.edu

E. A. Common

Department of Education, University of Michigan-Flint, Flint, MI, USA

\section{R. E. Hartzell}

Department of Disability and Psychoeducational Studies, University of Arizona, Tucson, AZ, USA in conducting rigorous research and dissemination during a phenomenal and unique time.

Understanding priorities for professional learning of educators, who are implementing the comprehensive, integrated, three-tiered (Ci3T; Lane et al., 2020) model of prevention is essential, as they address students' academic, behavioral and social emotional well-being needs. The special issue starts with an article by Common et al. examining the professional learning needs of faculty and staff from schools implementing Ci3T. In this study, the authors surveyed faculty and staff from 25 elementary schools in five districts across three states. The survey sought educators' views around the implementation of core components of their school's Ci3 T implementation and their preferences for professional learning content and modalities. The authors' results indicate that most respondents reported high levels of implementation for core Ci3T features across the tiers. In addition, educators reported high levels of implementation for 10 out of 19 research-based practices used within the tiered system. The top three areas for professional learning sought by educators in this sample were behavior deescalation techniques, small-group social skills instruction, and strategies for supporting students with internalizing behavior patterns. Finally, this study found top-rated avenues for professional learning, which included in-district, during-school workshops, courses for college credit on-line, teacher collaboratives/networks, and one-to-one coaching or mentoring. This study extends the literature on the professional learning needs to empower faculty and staff working within integrated tiered prevention models. 
Measuring fidelity through the course of the intervention is critical. Data informed decision making goes beyond student-level data (e.g., academic, behavior, social-emotional). Treatment integrity enables educators to make effective decisions about Tier 2 and 3 supports. In the next article, Buckman et al. examined the literature focusing on monitoring and reporting of treatment integrity within the context of tiered systems at Tier 1 (primary prevention). In this systematic review, the authors highlight not only the importance of treatment integrity as an indicator of trustworthiness in applied research (Cooper et al., 2020), but more so within the context of three-tiered models of prevention. Threetiered preventions require educators to monitor student responsiveness to Tier 1 prevention through multiple data sources-including treatment integrity data-to determine which students need additional support at higher intensity levels. The authors found more than $85 \%$ included studies reported procedures for treatment integrity and around $75 \%$ reported treatment integrity data for Tier 1 implementation. The most reported methods used were self-report and permanent product evaluation, and direct observation was least frequently reported as being used to monitor treatment integrity. This study extends the literature on the importance of monitoring and reporting treatment integrity in practice and research. In addition, the authors highlight the importance of contextualizing Tier 1 treatment integrity data as part of the data-informed decision-making process guiding Tier 2 and Tier 3 conversations.

In the third article, authors (Losinski, Thiele, Ennis, and Shaw) conducted a multiple-baseline design study across three $5^{\text {th }}$-grade student participants to examine the effects of self-regulated strategy development (SRSD) on correct answers in long division. Dependent variables were correct answers as well as rubric scores, which were more sensitive to the mathematical process. Students participated in a five-session intervention to learn the SRSD intervention (Harris \& Graham, 1999) and a partial quotients algorithm for solving longdivision problems. Treatment fidelity data showed the intervention agent (i.e., a special education doctoral student) implemented the intervention with very high levels of fidelity. All three students increased correct responses and rubric scores, demonstrating a functional relation between the intervention and long division outcomes. Students and the intervention agent rated the intervention as socially valid in terms of acceptability, understanding, feasibility, and school climate (Usage
Rating Profile Intervention Revised [URP-IR]; Chafouleas et al., 2011). Results indicate the intervention may be efficient, effective, and socially valid for classroom teachers and students.

In the fourth article, Sabey et al. presented an analysis of secondary data collected during an original study to examine the effect of precision request on the teacher's use of punishment (i.e., response cost and reprimands) and praise. This study was conducted with eight students with emotional disabilities in a self-contained 3rd5 th grade class in a suburban school. The dependent variables were the number of teacher reprimands, use of punishment, and use of praise. The independent variable was precision request that utilized a semi-scripted request format that included specific wording (i.e., "please" and "you need to") in a specific sequence, allowing for adequate wait time after requests, praise for compliance, and a punisher following noncompliant behavior. An A-B-A-B design was employed to determine the effectiveness of the intervention. The data suggested a strong positive functional relation between the teacher's use of precision request and praise. However, both reprimands and response cost did not show a strong relationship to decreased noncompliance, suggesting both did not function as punishment. The findings suggest that precision request may be a useful antecedent strategy, where students with EBD can benefit from formulating rules to promote desired behavior. However, future research is needed to determine if punishment is necessary or could be left out entirely, because it has the potential for producing negative side effects. In addition, although the precision request procedure was specifically designed to address students' compliance behaviors, it will be beneficial to evaluate its effectiveness with other relevant dependent variables beyond noncompliance, such as inattention, off-task, disruptive, or aggressive behavior.

In the last article, Beahm et al. investigated the resources that educators and other school personnel use to find information on effective behavior management strategies. In this mixed-methods study, the authors surveyed 238 educators in four West Virginia counties regarding the degree to which they used, trusted, could access, could implement, and could understand information regarding behavior management strategies. The authors evaluated six types of resources: search engines, internet media, professional organization websites, journals, colleagues, and professional development. In addition, the authors conducted a focus group with 10 
participants to share additional insights regarding why educators prefer specific resources and what they valued in them. The authors found that educators felt prepared to manage challenging student behavior and somewhat agreed that they had access to resources to support them. The majority of the respondents reported they used colleagues as their first behavior management resource, because they are familiar with their students and classrooms and share practices that can be trusted to be effective. In addition, colleagues are easy to access and understand, and provide trustworthy and usable information. Internet media received the lowest rating of all resources, despite $90 \%$ of participants indicating that they have downloaded and paid for resources from the internet. This study extends the literature by providing insights into where educators go for behavior management resources. It highlights their perceived use, trust in, accessibility to, implantability, and understanding of the variety and preferred features of behavior-management resources.

We sincerely appreciate the time and expertise of Claire St. Peter, editor-in-chief, for her guidance and advice. We are thankful to all the authors for their valuable contributions for advancing the knowledge base and research. We hope that their important work continues to help in developing a better understanding of complexities surrounding educational research and practices to improve outcomes for children and youth with and at risk for EBD. We are grateful to our reviewers for their constructive and informed critiques that were helpful in selecting the five manuscripts. We find the partnership between TECBD and the Council for Children with Behavioral Disorders (CCBD) extremely valuable, and extend an invitation to our readers to join us at the 2021 TECBD conference (http://tecbd. asu.edu). It is imperative for us to continue to build on the existing research and work with schools and other educational partners in developing better academic, emotional, behavioral, and mental health supports for children with EBD and their families.

\section{References}

Chafouleas, S. M., Briesch, A. M., Neugebauer, S. R., \& RileyTillman, T. C. (2011). Usage Rating Profile-Intervention (Revised). University of Connecticut.

Cooper, J. O., Heron, T. E., \& Heward, W. L. (2020). Applied behavior analysis. Pearson Education.

Harris, K. R., \& Graham, S. (1999). Programmatic intervention research: Illustrations from the evolution of self-regulated strategy development. Learning Disability Quarterly, 22, 251-262.

Lane, K. L., Menzies, H. M., Oakes, W. P., \& Kalberg, J. R. (2020). Developing a schoolwide framework to prevent and manage learning and behavior problems (2nd ed.). Guilford Press. 